\title{
Richard A. Settersten Jr, Frank F. Furstenberg Jr, and Rúben G. Rumbaut (eds), On the Frontier of Adulthood. Theory, Research, and Public Policy
}

The University of Chicago Press, Chicago, 2005, xvi + 591 pp

\author{
Aart C. Liefbroer
}

Published online: 13 March 2007

(C) Springer Science+Business Media B.V. 2007

On the Frontier of Adulthood is a collection of papers that chart the changes in the lives of American men and women roughly between the ages of 18 and 34. It brings together the work of a large number of renowned scholars in a huge volume (16 chapters, spanning more than 550 pages), producing, as the editors state, 'arguably the most thorough description of this period to date'. A central aim of the volume is to 'gain a more coherent and complete understanding of this period by focusing on the interplay of multiple transitions across multiple life domains (such as education, work, family, and leisure)'.

The book is made up of four parts. After an introduction by the editors, five chapters focus on the transition to adulthood in a comparative perspective, either by comparing the transition experiences of different US cohorts or by comparing the experience of US cohorts with those of cohorts in other countries. Part Three consists of eight chapters that study the transition to adulthood among recent US cohorts and focus on the roles played by institutions and family in launching children into adulthood, on racial and ethnic differences in the way this passage is made and on the consequences of different pathways into adulthood. The final part consists of two chapters that discuss policy issues related to the transition to adulthood.

I agree with the editors that this volume indeed gives the most thorough description of this period to date, with one big qualification: as far as the US is concerned. In fact, the amount of descriptive information is so overwhelming and diverse, that it is not easy to draw conclusions about how the frontier is changing. It would have been helpful to have a concluding chapter trying to synthesize the results of the empirical chapters. My own synthesis is that young adulthood in the US has become less standardized, and that many young adults (in particular blacks and members of other minorities) are facing

A. C. Liefbroer $(\square)$

Netherlands Interdisciplinary Demographic Institute, The Hague, The Netherlands e-mail: Liefbroer@nidi.nl 
increasing difficulties in making a successful transition in terms of achieving economic self-sufficiency, and stable and gratifying personal relationships.

Given the aim of the volume, a major challenge for the chapters is how to capture the interplay between events in several life domains, like education, work, union formation, and parenthood. There is huge diversity in how authors have gone about doing so. Two issues seem particularly important in this respect. A first issue is whether to use statistical methods to classify respondents or not. In several papers, latent class analysis is used to differentiate between young adults on the basis of information on their educational, occupational, and family life situation. In some other papers, classifications are derived without the help of a statistical method, but based either on theoretical or practical considerations. The first approach has the potential drawback that it might be hard to correctly interpret the empirically derived classes, whereas the second approach has the potential disadvantage that one overlooks empirical distinctions that do not fit into one's theoretical scheme. A second issue deals with whether to have a cross-sectional 'snapshot' approach to classifying young adults or a longitudinal 'trajectory' approach. Most chapters classify young adults based on their position in several life domains at a certain age, even if information on sequencing and timing of life events is available, thus ignoring the dynamic character of the data. Just a few chapters try to classify young adults based on the sequence of events during young adulthood or the sequence and the timing of events. However, these chapters differ strongly in the way the data is handled. In one, a descriptive analysis of marriage and childbearing histories is presented; in another, an adhoc classification of life courses is presented; in a third, a statistical method (i.c., a monothetic divisive algorithm) is applied. Although it is laudable that all of these chapters focus on the transition to adulthood in multiple life domains, many do not exploit the full range of possibilities that the dynamic character of the data allow. This suggests that a lot of headway is still to be made in charting the trajectories of young adults across multiple life domains. The chapters in this volume present us with a rich variety of possible approaches to build upon, but do not yet offer definite answers of how to proceed in studying the multiple pathways into adulthood.

Although my comments will have made it clear that I do not feel that $O n$ the Frontier of Adulthood completely lives up to its promises quoted at the beginning of this review, I do feel that this volume is really at the frontier of research on young adulthood and makes a very important contribution to the literature on the transition to adulthood. As such, it is compulsory reading for anyone with a serious interest in this life phase. 